\title{
PLANAR TESSELLATIONS THAT HAVE THE HALF-GILBERT STRUCTURE
}

\author{
JAMES BURRIDGE*, RICHARD COWAN**
}

\author{
* Department of Mathematics, University of Portsmouth, Portsmouth, UK. james.burridge@port.ac.uk \\ $*^{*}$ School of Mathematics and Statistics, University of Sydney, 2006, NSW, Australia. richard.cowan@sydney.edu.au
}

\begin{abstract}
In the full rectangular version of Gilbert's planar tessellation $[1,2,3]$, lines extend either horizontally (with east- and west-growing rays) or vertically (north- and south-growing rays) from seed points which form a stationary Poisson point process, each ray stopping when it meets another ray that has blocked its path. In the half-Gilbert rectangular version, east and south growing rays, whilst having the potential to block each other, do not interact with west and north rays, and vice versa. East and south growing rays have a reciprocality of blocking, as do west and north. In this paper we significantly expand and simplify the half-Gilbert analytic results that we gave in [3]. We also show how the idea of reciprocality of blocking between rays can be used in a much wider context, with rays not necessarily orthogonal and with seeds that produce more than two rays.
\end{abstract}

Keywords: Random Tessellation; point process; crack formation; division of space 2010 Mathematics Subject Classification: Primary 60D05; 05B45 Secondary 60G55; 51M20

\section{Our improved RESUlts in the ReCtAngular CASE}

Consider a stationary Poisson seed process of intensity $\lambda$ in the plane, with seeds independently marked either $H$ (growing horizontally) with probability $q$ or $V$ (growing vertically) with probability $1-q$. Commencing simultaneously at time zero with a common and constant growthrate, each seed produces two rays in directions matching their label $(H$ or $V)$. For example, an $H$-seed grows one ray toward the east and another toward west. A ray ends its growth when its growing tip meets a ray whose direction gives it reciprocality of blocking. An east-growing ray (E-ray) has this reciprocality with south-growing rays ( $S$-rays) and each can block the other. A similar relationship exists between north- and west-growing rays. There is no blocking, however, of $E$-rays by $N$-rays (and vice versa) or of $S$-rays by $W$-rays (and vice versa). Naturally, a ray cannot be blocked by a ray whose growth path is parallel to itself; for example, an $E$-ray Date: August 25, 2015. 
won't be blocked by other $E$-rays or by $W$-rays. A realisation of the rectangular half-Gilbert tessellation having $q=\frac{1}{2}$ is shown in Figure $1(\mathrm{a})$.

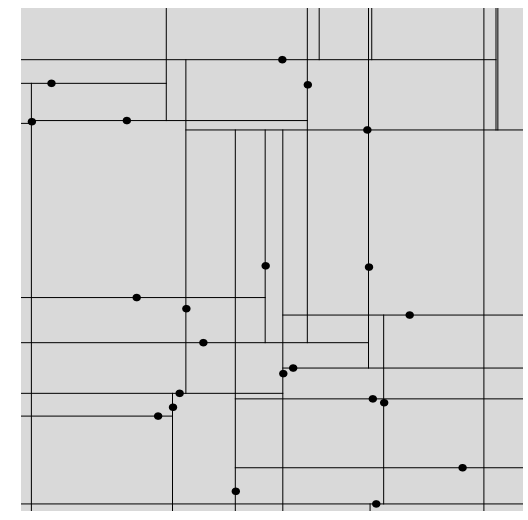

(a)

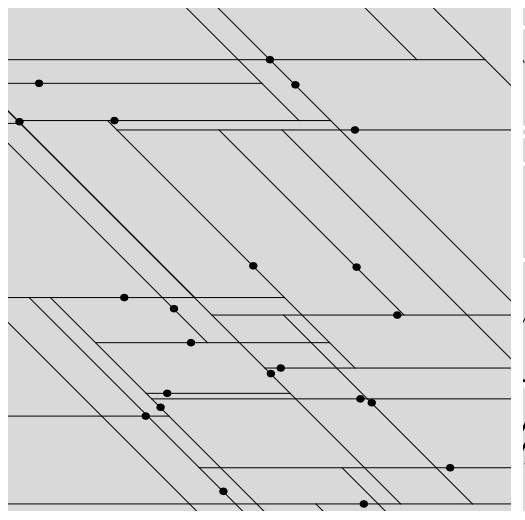

(b)

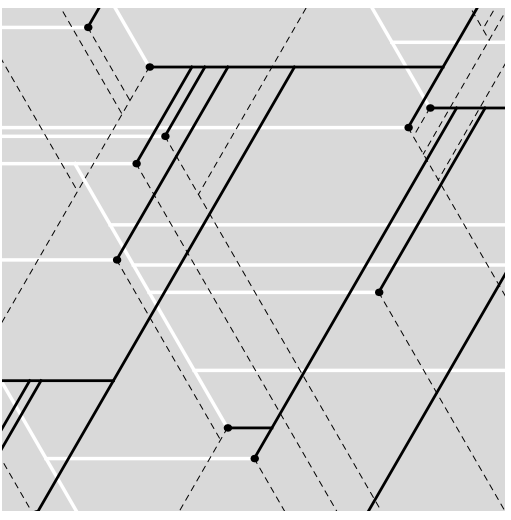

(c)

FIGURE 1. (a) The half-Gilbert rectangular-celled tessellation with $q=\frac{1}{2}$. (b) The seed positions and $q$ value are as in (a); those seeds in (a) which produce vertical seeds now produce rays that grow North-West and South-East. The East growing rays block, and are blocked by, the South-East growing rays. Likewise the $W$-rays and $N W$-rays have reciprocal blocking. Note that there are many changes, from the situation in (a), in the blocking decisions and the order in which a ray encounters other rays. (c) Every seed has three emanating rays with three different shading patterns (either white, thick black or dashed black). There are two types of seed, one where the horizontal ray grows east and in the other type it grows west. The probability that a seed is of the former type is $q$ (here $q=\frac{1}{3}$ ). Rays of the same shading pattern have reciprocal blocking.

Before the half-Gilbert model was introduced in [3], no tessellation with a Gilbert-style blocking mechanism had yielded any exact mathematical results. So the half-Gilbert model has some importance, we think; whilst it doesn't model a physical process, it does enrich the theory of tessellations, because very few models within that theory have analytic tractability. In one small respect, too, there is greater stochastic interest in the 'half-model' as it has both + and T-style vertices (see Figure 1(a)) whereas the full-Gilbert model has only the $T$ style. Additionally it is demonstrated in [3] that the distributions of ray length in the rectangular full- and half-Gilbert models are almost identical when the seed intensity in the former model is half that of the latter. This gives another approximating role for the half-Gilbert.

One aim in our work has been the investigation of the random variable $L_{E}$ defined as the final length of a typical stopped E-ray. Although we focus on east-growing rays, the results for $L_{E}$ apply to the vertical rays, by merely replacing $q$ with $1-q$, and to the $W$-rays because the final lengths $L_{E}$ and $L_{W}$ of typical east and west rays are identically distributed. 
In our earlier paper [3], we were only able to find simple formulae in the balanced case, $q=\frac{1}{2}$. These (stated for E-rays) were

$$
\mathbb{E}\left(L_{E}\right)=\frac{\pi}{\sqrt{\lambda}\left(\Gamma\left(\frac{3}{4}\right)\right)^{2}}
$$

and a formula for $\mathbb{E}\left(L_{E}^{2}\right)$ which involved an integral. The same expressions held for the other compass directions. A extremely unwieldy expression for $\mathbb{E}\left(L_{E}\right)$ when $q$ is general was also given in $[3]$.

In the current paper we improve these results to give many moments of $L_{E}$ and the Laplace transform $\mathcal{L}_{t}:=\mathbb{E}\left(e^{-t L_{E}}\right)$ which can generate as many moments as one desires. Our main result, proved in the next section, is the following theorem.

Theorem: For general $q \in(0,1)$,

$$
\mathcal{L}_{t}:=\mathbb{E}\left(e^{-t L_{E}}\right)=1-t \sqrt{\frac{2}{\lambda}} \frac{H_{q-1}\left(\frac{t}{\sqrt{2 \lambda}}\right)}{H_{q}\left(\frac{t}{\sqrt{2 \lambda}}\right)},
$$

with the same expression for $W$-rays. For $N$-rays and $S$-rays, the formula has $q$ replaced by $1-q$. Here $H_{\nu}(\cdot)$ is a generalisation of the Hermite polynomial to non-integer $\nu$ (see (??) and [6]).

Moments of $L_{E}$ are found easily from the Theorem.

Corollary: We define the ratio $\boldsymbol{\psi}:=\Gamma\left(\frac{1}{2}-\frac{q}{2}\right) / \Gamma\left(1-\frac{q}{2}\right)$ of Gamma functions. The following moments are polynomials in $\boldsymbol{\psi}$.

$$
\begin{array}{ll}
\mathbb{E}\left(L_{E}\right)=\frac{\boldsymbol{\psi}}{\sqrt{2 \lambda}} ; & \mathbb{E}\left(L_{E}^{3}\right)=\frac{3 \boldsymbol{\psi}}{\lambda \sqrt{2 \lambda}}\left(1+2 q+q^{2} \boldsymbol{\psi}^{2}\right) ; \\
\mathbb{E}\left(L_{E}^{2}\right)=\frac{1}{\lambda}\left(2+q \boldsymbol{\psi}^{2}\right) ; & \mathbb{E}\left(L_{E}^{4}\right)=\frac{2}{\lambda^{2}}\left(4+4 q+4 q(1+2 q) \boldsymbol{\psi}^{2}+3 q^{3} \boldsymbol{\psi}^{4}\right) .
\end{array}
$$

Note that $\boldsymbol{\psi}$ can be rearranged in many ways using the identities $\sqrt{\pi} \Gamma(2 z)=2^{2 z-1} \Gamma(z) \Gamma\left(z+\frac{1}{2}\right)$ and $\Gamma(z) \Gamma(1-z)=\pi / \sin (\pi z)$. The consistency of (1) with the Corollary follows from the second of these identities.

The same results apply for $W$-rays and, with $q$ replaced by $(1-q)$, to the vertical rays. To get the moments for a typical ray's length (taking all directions of growth into account) we require weighted averages: for example, the expected length $L$ of a typical ray is $q \mathbb{E}\left(L_{E}\right)+(1-q) \mathbb{E}\left(L_{S}\right)$ 
which equals $\left(q \boldsymbol{\psi}_{q}+(1-q) \boldsymbol{\psi}_{1-q}\right) / \sqrt{2 \lambda}$; here the dependence of $\boldsymbol{\psi}$ on $q$ is emphasised by the added subscript.

Plan OF The PAPER: As indicated in our abstract, we have other new ideas to present. These come firstly from modifying the angle between the two systems of parallel rays, breaking from the orthogonality that we see in the half-Gilbert rectangular model. Some effects of this are shown in Figure 1(b) where the cells are now parallelograms and where rays block and are blocked by other rays which create an angle of $\alpha$ (which is $45^{\circ}$ in this figure); E-growing and $S E$-growing form a reciprocal-blocking pair as do $W$-growing and $N W$-growing pair.

In the rectangular case, $\alpha=\pi / 2$. We shall see in Section 3 that the results change from the rectangular case in a simple way; $\lambda$ is replaced by $\lambda^{*}:=\lambda \sin (\alpha)$ in the formulae for the rectangular half-Gilbert model, for example in the Theorem and its Corollary. Secondly, we develop a class of models where rays can grow in $2 m$ different directions, these directions being grouped to give $m$ pairs each operating with reciprocal blocking. They too can be analysed by simple changes to (and amalgamations of) the rectangular formulae. Thirdly, seeds whose two rays are not collinear are considered as are seeds which grow more than two rays.

These new developments will be set out in Section 3, where we shall see that at their heart lies a trapezoidal stopping set process, originally introduced in the analysis of the rectangular half-Gilbert model [3], and described in greater detail and generality in Section 2 below. Readers seeking informal ideas rather than formal analysis could perhaps go to Section 3 first.

\section{Proof of the Theorem and its Corollary}

Trapezoidal Stopping Set Process: Our analytical results here are obtained through the analysis of Markov stopping set processes $[4,5]$. The archetypal process for the analysis of tessellations with reciprocal blocking rules arises in the analysis of the rectangular half-Gilbert model, and involves trapezia. An example is shown in Figure 2(a).

The process begins with the expansion of a trapezoidal set into an unbounded domain lying between the lines $y=0, x=0$ and $y=h+x$ where $h:=|Z A|$ is the length of the left edge of the expanding set. The unbounded domain is called the live zone and is populated by the stationary Poisson seed process described in Section 1. Upon meeting the first seed (point $C$ in the Figure), the frontier of the expanding set stops, creating a trapezium $\mathrm{S}_{1}$, whose area is an exponential random variable with parameter $\lambda$. If the stopping seed is $V$-type then the process ends; if not a new trapezoidal set having left boundary of length $|B C|$ begins growth. If the first 


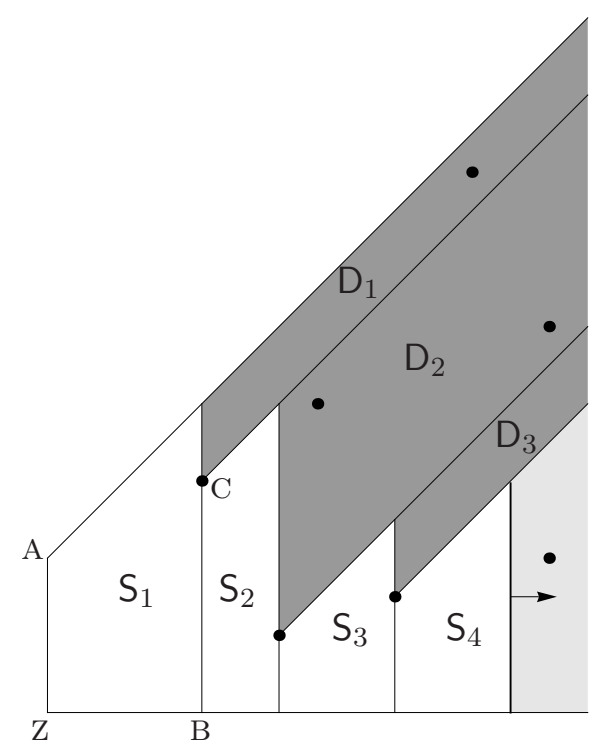

(a)

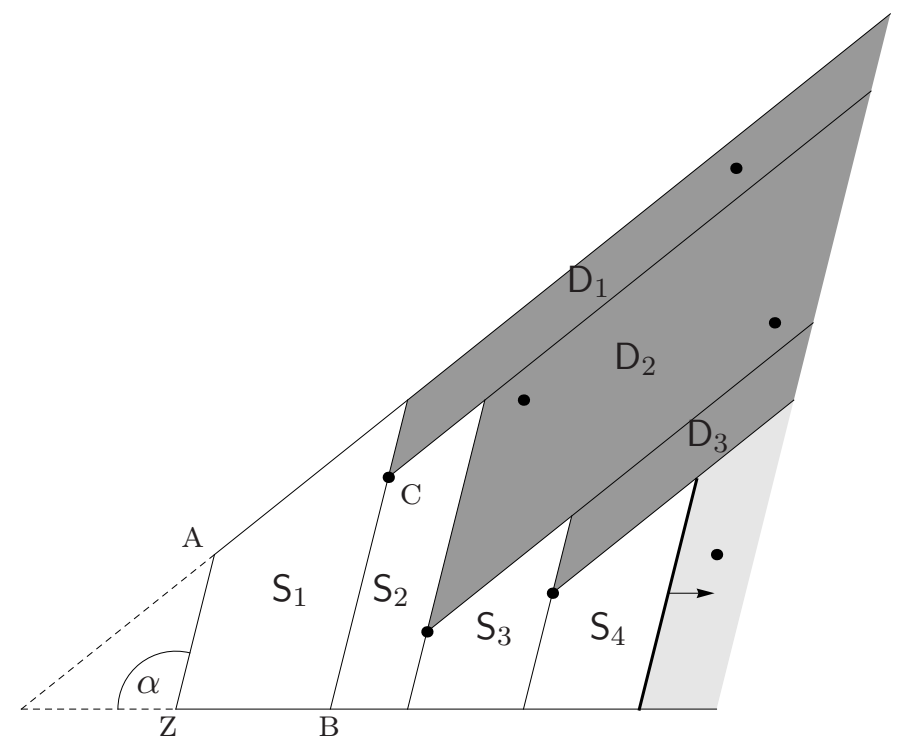

(b)

FIGURE 2. (a) Trapezoidal stopping sets $S_{1}, S_{2}, \ldots$ and dead zones $D_{1}, D_{2} \ldots$ in the rectangular half-Gilbert model. The final trapezoidal set with bold right frontier is still growing in the direction of the arrow, and will shortly be stopped by the seed (which happens to be a $V$-seed) in the light grey Live Zone. The Dead Zones are shaded dark grey. (b) This picture replaces (a) when the two systems of growing rays are not orthogonal, that is, when $\alpha \neq \pi / 2$.

set is stopped by an $H$-type then a dead zone, $\mathrm{D}_{1}$, is created, as shown in Figure 2(a). Once this zone is removed from consideration we are left with a new live zone which, apart from the length of its left boundary, provides a stochastic situation identical to the original. Trapezoidal sets $S_{2}, S_{3}, \ldots$ and dead zones $D_{2}, D_{3}, \ldots$ continue to be formed until the process is stopped by a $V$-type seed. We let the sum of the bases of these trapezia define a random variable $X$.

The connection between the stopping set process and the underlying ray process may be seen by noting that if only the blocking effects of rays originating within the live zone are considered, then the criterion for the process to end is that the ray from a $V$-type seed crosses the $x$ axis. The zones $\mathrm{D}_{k}$ are dead in the sense that the seeds within them cannot influence the point at which the $x$ axis is first intersected. In the case where the initial live zone has $h=0, \mathrm{~S}_{1}$ is an isosceles triangle, and rays emanating from outside the live zone can have no influence on the point at which the $x$ axis is first intersected by a vertical ray. In this case $X$ is identically distributed to the distance, $L_{E}$, travelled by a horizontal ray emerging from a seed located at the origin, before it is blocked.

A ReCURSIVE EQUATION FOR THE Distribution of $X$ : The self-similar structure of the sequence of stopping sets allows us to construct a recursive equation for the distribution of $X$. 
Letting $y$ denote the length of the left boundary of a generic set, $\mathrm{S}_{n}$, in the sequence, it follows from the exponential distribution of $\mathrm{S}_{n}$ 's area that its base $R$ will have distribution:

$$
\mathbb{P}(R \in(r, r+d r])=\lambda(r+y) e^{-\frac{\lambda}{2}\left(r^{2}+2 r y\right)} d r .
$$

If the stopping seed for set $\mathrm{S}_{n}$ is $V$-type then the process ends. Otherwise another dead zone, $\mathrm{D}_{n}$, is created and further trapezoidal stopping sets are formed until a $V$-type is met. The density function of $X$, conditional on $y$ will therefore be:

$$
g(x \mid y)=(1-q) \lambda(x+y) e^{-\frac{\lambda}{2}\left(x^{2}+2 x y\right)}+q \lambda \int_{0}^{\infty} e^{-\frac{\lambda}{2}\left(r^{2}+2 r y\right)}\left[\int_{0}^{r+y} g(x-r \mid u) d u\right] d r
$$

where $g(x \mid y)=0$ if $x<0$. The first term on the right accounts for the case where the first seed is $V$-type, and the second term for the case where it is $H$-type and the process is effectively restarted with a different left boundary condition having already covered some horizontal distance. Note that the probability density function for ray length $L_{E}$ is $g(x \mid 0)$.

The Laplace Transform: We define the Laplace Transform of the conditional probability density function $g(\cdot \mid y)$ :

$$
\mathcal{L}_{t}(y)=\int_{0}^{\infty} e^{-t x} g(x \mid y) d x .
$$

Here the general value of $q \in(0,1)$ is implicit.

Lemma 1: For $q \in(0,1), \mathcal{L}_{t}(\cdot)$ satisfies the following second-order differential equation.

$$
\frac{d^{2} \mathcal{L}_{t}(y)}{d y^{2}}-(\lambda y+t) \frac{d \mathcal{L}_{t}(y)}{d y}-\lambda(1-q) \mathcal{L}_{t}(y)=-\lambda(1-q)
$$

The equation is a generalisation of Hermite's equation.

Proof of Lemma 1: From equation $(3), \mathcal{L}_{t}(y)$ satisfies

$$
\begin{aligned}
\mathcal{L}_{t}(y)=(1-q) \lambda \int_{0}^{\infty}(x+y) e^{-\frac{\lambda}{2}\left(x^{2}+2 x y\right)-t x} d x & \\
& +q \lambda \int_{0}^{\infty} e^{-\frac{\lambda}{2}\left(r^{2}+2 r y\right)}\left[\int_{0}^{r+y}\left(\int_{0}^{\infty} e^{-t x} g(x-r \mid u) d x\right) d u\right] d r .
\end{aligned}
$$

The second term in this equation may be expressed in terms of $\mathcal{L}_{t}(\cdot)$ by noting that

$$
\begin{aligned}
\int_{0}^{\infty} e^{-t x} g(x-r \mid u) d x & =\int_{0}^{\infty} e^{-t(z+r)} g(z \mid u) d z \\
& =e^{-t r} \mathcal{L}_{t}(u),
\end{aligned}
$$


giving an integral equation for $\mathcal{L}_{t}(\cdot)$. This equation converts into a differential equation with independent variable $y$. We begin by simplifying the second term in equation (5) using integration by parts.

$$
\begin{aligned}
\int_{0}^{\infty} \lambda e^{-\frac{\lambda}{2}\left(r^{2}+2 r\left(y+\frac{t}{\lambda}\right)\right)}\left[\int_{0}^{r+y} \mathcal{L}_{t}(u) d u\right] d r= \\
\sqrt{\frac{\pi \lambda}{2} e^{\frac{(y \lambda+t)^{2}}{2 \lambda}}}\left[\operatorname{erfc}\left(\frac{y \lambda+t}{\sqrt{2 \lambda}}\right) \int_{0}^{y} \mathcal{L}_{t}(u) d u+\int_{y}^{\infty} \operatorname{erfc}\left(\frac{u \lambda+t}{\sqrt{2 \lambda}}\right) \mathcal{L}_{t}(u) d u\right]
\end{aligned}
$$

Evaluating the integral in the first term of (5), and then combining both terms, we obtain

$$
\begin{aligned}
& \mathcal{L}_{t}(y)=(1-q) {\left[1-\sqrt{\frac{\pi}{2 \lambda}} t e^{\frac{(y \lambda+t)^{2}}{2 \lambda}} \operatorname{erfc}\left(\frac{y \lambda+t}{\sqrt{2 \lambda}}\right)\right] } \\
&+q \sqrt{\frac{\pi \lambda}{2}} e^{\frac{(y \lambda+t)^{2}}{2 \lambda}}\left[\operatorname{erfc}\left(\frac{y \lambda+t}{\sqrt{2 \lambda}}\right) \int_{0}^{y} \mathcal{L}_{t}(u) d u+\int_{y}^{\infty} \operatorname{erfc}\left(\frac{u \lambda+t}{\sqrt{2 \lambda}}\right) \mathcal{L}_{t}(u) d u\right]
\end{aligned}
$$

Dividing by $\sqrt{\pi \lambda / 2} \exp \left[(y \lambda+t)^{2} /(2 \lambda)\right]$, differentiating with respect to $y$, and then multiplying by the same factor again we have

$$
\frac{d \mathcal{L}_{t}(y)}{d y}-(t+\lambda y) \mathcal{L}_{t}(y)=-(1-q) \lambda y-q \lambda \int_{0}^{y} \mathcal{L}_{t}(u) d u
$$

A further differentiation with respect to $y$ yields the result of Lemma 1, thereby completing the proof of this lemma.

Generalised Hermite equation: The solution to this differential equation is known. The general solution, given by the Mathematica software and provable by substitution in (4), is

$$
\mathcal{L}_{t}(y)=1+c_{1}(t) H_{q-1}\left(\frac{y \lambda+t}{\sqrt{2 \lambda}}\right)+c_{2}(t){ }_{1} F_{1}\left(\frac{1-q}{2}, \frac{1}{2},\left(\frac{y \lambda+t}{\sqrt{2 \lambda}}\right)^{2}\right)
$$

where $c_{1}(\cdot)$ and $c_{2}(\cdot)$ are general 'constants' of integration not dependent on $y$ (but, as shown, possibly dependent on $t$ ). The coefficient of $c_{2}(t)$ in (7) is an example of a Kummer confluent hypergeometric function. Because all the $t$ derivatives of this function, evaluated at $t=0$, diverge as $y \rightarrow \infty$, we may discard it from the solution (that is, we can set $c_{2}(t)=0$ ) on the grounds that for all $x>0, \lim _{y \rightarrow \infty} g(x \mid y)$ should equal zero. The coefficient of $c_{1}(t)$, a generalisation of the Hermite polynomials $H_{n}(\cdot)$ to non-integer $n$, has the correct asymptotic behaviour as $y \rightarrow \infty$. It is a combination of Kummer functions [6].

$$
H_{\nu}(z)=\sqrt{\pi} 2^{\nu}\left(\frac{{ }_{1} F_{1}\left(-\frac{\nu}{2} ; \frac{1}{2} ; z^{2}\right)}{\Gamma\left(\frac{1-\nu}{2}\right)}-\frac{2 z_{1} F_{1}\left(\frac{1-\nu}{2} ; \frac{3}{2} ; z^{2}\right)}{\Gamma\left(-\frac{\nu}{2}\right)}\right),
$$


where ${ }_{1} F_{1}(a, b, z):=1+\sum_{j=1}^{\infty} r_{j} z^{j} / j$ ! with

$$
r_{j}:=\frac{a(a+1)(a+2) \ldots(a+j-1)}{b(b+1)(b+2) \ldots(b+j-1)}, \quad j \geq 1 .
$$

These special functions have interesting properties; for later use, we especially note the identity

$$
H_{\nu}(z)+\frac{1}{2(\nu+1)} H_{\nu+2}(z)=\frac{z}{\nu+1} H_{\nu+1}(z)
$$

valid for general $\nu$ and easily proved by calculating the coefficients of $z^{2 n}$ and $z^{2 n+1}$ in series expansions of both sides in (8).

It remains to evaluate the 'constant' $c_{1}(t)$.

Lemma 2: The function $c_{1}(\cdot)$ is given by the following formula.

$$
c_{1}(t)=\frac{-t \sqrt{2}}{\sqrt{\lambda} H_{q}\left(\frac{t}{\sqrt{2 \lambda}}\right)} .
$$

Proof of Lemma 2: We substitute the new function $\mathcal{L}_{t}(\cdot)$ given in $(7)$ (now with $c_{2}(t)=0$ ) into the integral equation (6), evaluated at $y=0$. This yields an equation for $c_{1}(t)$.

$$
\begin{aligned}
& 1+c_{1}(t) H_{q-1}\left(\frac{t}{\sqrt{2 \lambda}}\right)=\mathcal{L}_{t}(0)=(1-q)\left[1-\sqrt{\frac{\pi}{2 \lambda}} t e^{\frac{t^{2}}{2 \lambda}} \operatorname{erfc}\left(\frac{t}{\sqrt{2 \lambda}}\right)\right] \\
&+q \sqrt{\frac{\pi \lambda}{2}} e^{\frac{t^{2}}{2 \lambda}} \int_{0}^{\infty} \operatorname{erfc}\left(\frac{u \lambda+t}{\sqrt{2 \lambda}}\right)\left[1+c_{1}(t) H_{q-1}\left(\frac{u \lambda+t}{\sqrt{2 \lambda}}\right)\right] d u
\end{aligned}
$$

Using a change of variable, $z=(u \lambda+t) / \sqrt{2 \lambda}$, the integral on the right-hand side breaks up into two integrals which simplify (using Mathematica, which works better with $1-\operatorname{erf}(\cdot)$ rather than $\operatorname{erfc}(\cdot)$ in this context), as follows.

$$
\begin{aligned}
& \int_{0}^{\infty} \operatorname{erfc}\left(\frac{u \lambda+t}{\sqrt{2 \lambda}}\right) d u+c_{1}(t) \int_{0}^{\infty} \operatorname{erfc}\left(\frac{u \lambda+t}{\sqrt{2 \lambda}}\right) H_{q-1}\left(\frac{u \lambda+t}{\sqrt{2 \lambda}}\right) d u \\
= & \sqrt{\frac{2}{\lambda}} \int_{t / \sqrt{2 \lambda}}^{\infty} \operatorname{erfc}(z) d z+c_{1}(t) \sqrt{\frac{2}{\lambda}} \int_{t / \sqrt{2 \lambda}}^{\infty} \operatorname{erfc}(z) H_{q-1}(z) d z \\
= & \frac{\sqrt{2}}{\sqrt{\pi \lambda}} e^{-\frac{t^{2}}{2 \lambda}}-\frac{t}{\lambda} \operatorname{erfc}\left(\frac{t}{\sqrt{2 \lambda}}\right)+\frac{c_{1}(t)}{q \sqrt{2 \pi \lambda}}\left[2 e^{-\frac{t^{2}}{2 \lambda}} H_{q-1}\left(\frac{t}{\sqrt{2 \lambda}}\right)-\sqrt{\pi} \operatorname{erfc}\left(\frac{t}{\sqrt{2 \lambda}}\right) H_{q}\left(\frac{t}{\sqrt{2 \lambda}}\right)\right] .
\end{aligned}
$$

The right-hand side of (9) then becomes

$$
1-\sqrt{\frac{\pi}{2 \lambda}} t e^{\frac{t^{2}}{2 \lambda}} \operatorname{erfc}\left(\frac{t}{\sqrt{2 \lambda}}\right)+c_{1}(t)\left[H_{q-1}\left(\frac{t}{\sqrt{2 \lambda}}\right)-\frac{\sqrt{\pi}}{2} e^{\frac{t^{2}}{2 \lambda}} \operatorname{erfc}\left(\frac{t}{\sqrt{2 \lambda}}\right) H_{q}\left(\frac{t}{\sqrt{2 \lambda}}\right)\right]
$$


and when equated to the left-hand side of (9), Lemma 2 is proven.

Remark: If Mathematica is unavailable, integration by parts then application of the identity (8) resolves the difficult integral in the proof above.

$$
\begin{aligned}
\int_{t / \sqrt{2 \lambda}}^{\infty} \operatorname{erfc}(z) H_{q-1}(z) d z & =\frac{1}{q \sqrt{2 \lambda}} \int_{z=t / \sqrt{2 \lambda}}^{\infty} \operatorname{erfc}(z) d\left[H_{q}(z)\right] \\
& =\frac{1}{q \sqrt{2 \pi \lambda}}\left[-\sqrt{\pi} \operatorname{erfc}\left(\frac{t}{\sqrt{2 \lambda}}\right) H_{q}\left(\frac{t}{\sqrt{2 \lambda}}\right)+2 \int_{t / \sqrt{2 \lambda}}^{\infty} e^{-z^{2}} H_{q}(z) d z\right] .
\end{aligned}
$$

Note that $\frac{d}{d z}\left[e^{-z^{2}} H_{q-1}(z)\right]=-e^{-z^{2}} H_{q}(z)$; direct differentiation and the use of (8) establishes this result. The last integral can now be evaluated.

The Complete solution: Lemmas 1 and 2, combined with other analysis of this section, show that

$$
\mathcal{L}_{t}(y)=1-t \sqrt{\frac{2}{\lambda}} \frac{H_{q-1}\left(\frac{y \lambda+t}{\sqrt{2 \lambda}}\right)}{H_{q}\left(\frac{t}{\sqrt{2 \lambda}}\right)} .
$$

Setting $y=0$ proves the Theorem given in Section 1. The moment results in the Corollary to the theorem are easily found from the Laplace Transform. Equation (10) also allows us to show that, in the context of Figure 2(a), the conditional expectation of the random variable $X$ given $y$ is

$$
2^{-q} \sqrt{\frac{2}{\lambda \pi}} \Gamma\left(\frac{1}{2}-\frac{q}{2}\right) H_{q-1}\left(\frac{y \sqrt{\lambda}}{\sqrt{2}}\right)
$$

which tends to zero as $y \rightarrow \infty$, if $q \in(0,1)$.

\section{Other MOdELS USING RECIPROCAL BLOCKING RULES}

The Simplest generalisation: Consider the model depicted in Figure 1(b). It will play a generic role for us. The only change from the basic model analysed in Section 2 is the shift from $\alpha=90^{\circ}$ to a general value of $\alpha \in\left(0,180^{\circ}\right)$. Here $\alpha$ is defined as follows: if $s_{1}$ and $s_{2}$ are the positions of two seeds and if $b$ is the point where one seed's ray blocks the ray of the other seed, then $\alpha$ is defined as the angle $s_{1} b s_{2}$. Figure 1(b) shows the case $\alpha=45^{\circ}$.

The intensity of seeds is still denoted by $\lambda$, we still have two types of seed (each with two emanating rays), a proportion $q$ of seeds grow horizontally and the growth of each seed's rays is bidirectional with the two directions being collinear (or, one might say, 'opposing').

A simple Analysis of This Generic $\alpha$-PROBlem: The argument in Section 2 uses the stopping sets shown in Figure 2(a). The generic problem with general $\alpha$ has an identical argument, 
except that Figure 2(b) is used instead. Simple trigonometric adjustments in the argument yield a new version for (2), namely

$$
\begin{aligned}
\mathbb{P}(R \in(r, r+d r]) & =\lambda(r+y) \sin \alpha e^{-\frac{\lambda}{2}\left(r^{2}+2 r y\right) \sin \alpha} d r \\
& =\lambda^{*}(r+y) e^{-\frac{\lambda^{*}}{2}\left(r^{2}+2 r y\right)},
\end{aligned}
$$

where $\lambda^{*}:=\lambda \sin \alpha$. Likewise the new version of (3) has the same simple replacement:

$$
\begin{aligned}
g(x \mid y)= & (1-q) \lambda(x+y) \sin \alpha e^{-\frac{\lambda}{2}\left(x^{2}+2 x y\right) \sin \alpha} \\
& +q \lambda \sin \alpha \int_{0}^{\infty} e^{-\frac{\lambda}{2}\left(r^{2}+2 r y\right) \sin \alpha}\left[\int_{0}^{r+y} g(x-r \mid u) d u\right] d r \\
= & (1-q) \lambda^{*}(x+y) e^{-\frac{\lambda^{*}}{2}\left(x^{2}+2 x y\right)}+q \lambda^{*} \int_{0}^{\infty} e^{-\frac{\lambda^{*}}{2}\left(r^{2}+2 r y\right)}\left[\int_{0}^{r+y} g(x-r \mid u) d u\right] d r,
\end{aligned}
$$

where $g(x \mid y)=0$ if $x<0$.

It is important to note that these new versions can be found from the old versions by replacing $\lambda$ with $\lambda^{*}:=\lambda \sin \alpha$. So all of the analysis of Section 2 remains valid with this replacement. Therefore we have proved the following lemma - a result which provides a foundation for the remainder of this section.

Lemma 3: For general $\alpha$, our main results given in the Theorem and its Corollary hold for the reciprocal-pair of rays in the generic problem, provided $\lambda$ is replaced by $\lambda^{*}:=\lambda \sin \alpha$.

More than Two TYPES OF SEED: Consider now models, such as the two illustrated in Figures 3(a) and 3(b), which have $m$ types of seed, where $m>2$. The figures, in which $m=3$, have type-1 seeds growing opposing horizontal rays east and west, type- 2 growing rays heading at angles of $60^{\circ}$ and $\left(60^{\circ}+180^{\circ}\right)=240^{\circ}$ measured anti-clockwise from the positive $x$-axis whilst the third type sends its rays out with these angles being $120^{\circ}$ and $\left(120^{\circ}+180^{\circ}\right)=300^{\circ}$. (The $E$-rays and $W$-rays are named $0^{\circ}$ and $180^{\circ}$ respectively in this labelling scheme.) The proportion of type $j$ is denoted by $q_{j}$. For both Figures $3(\mathrm{a})$ and $3(\mathrm{~b}), q_{j}=\frac{1}{3}, j=1,2,3$. There are many ways to pair off the six directions; the choices shown in the figures are defined in the caption.

We note that each reciprocating pair of directions can be studied separately, because the different pairs present independent processes when viewed within the generic format given in Figure 2(b).

Let us focus on a reciprocal pair which involves rays (whose length we seek) from a type- $i$ seed blocked by rays from a type- $j$ seed. If $\lambda$ denotes, as before, the intensity of all seeds, 


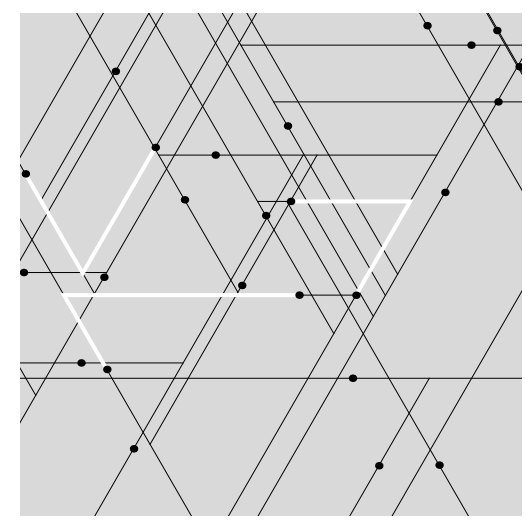

(a)

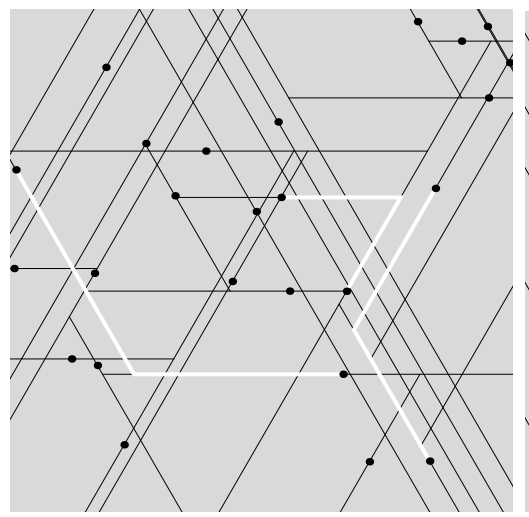

(b)

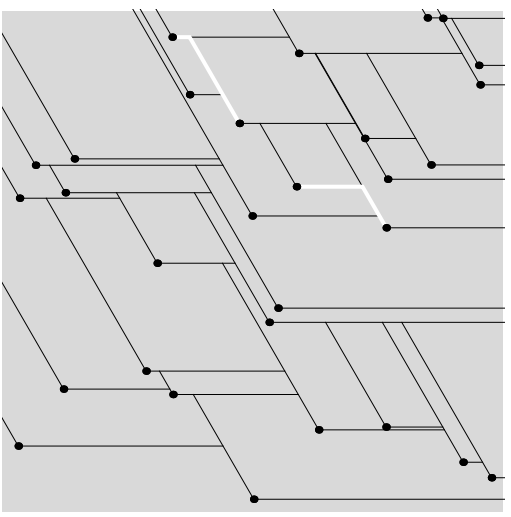

(c)

FIGURE 3. (a) and (b) Half-Gilbert tessellation based on three orientations of seed growth: opposing rays at angles $0^{\circ}$ and $180^{\circ}$; opposing rays at angles $60^{\circ}$ and $240^{\circ}$; opposing rays at angles $120^{\circ}$ and $300^{\circ}$. All these angles are measured in the usual way (anti-clockwise from the positive $x$-axis). Orientations are independent and equally prevalent (see text). Examples of the angle $\alpha$, defined in the text, are highlighted in white. (a) The reciprocality of blocking rules pair $0^{\circ}$ rays with $60^{\circ}$ rays, $120^{\circ}$ with $180^{\circ}$ and $240^{\circ}$ with $300^{\circ}$. All $\alpha$ angles equal $60^{\circ}$. (b) Here the pairing rules are $0^{\circ}$ with $60^{\circ}, 120^{\circ}$ with $240^{\circ}$ and $180^{\circ}$ with $300^{\circ}$. Two of the $\alpha$ angles equal $120^{\circ}$; one is $60^{\circ}$. (c) There is only one type of seed. It produces an east ray and a ray of angle $120^{\circ}$. The east rays $\left(0^{\circ}\right)$ are blocked by the $120^{\circ}$ rays and vice versa. Non convex cells can occur.

then the total intensity of type- $i$ and type- $j$ is $\lambda\left(q_{i}+q_{j}\right)$ whilst the proportion of these two types that are type- $i$ is $q_{i} /\left(q_{i}+q_{j}\right)$. So, using the relevant $\alpha$, the generic analysis proceeds with $\lambda^{*}=\lambda\left(q_{i}+q_{j}\right) \sin \alpha$ and $q=q_{i} /\left(q_{i}+q_{j}\right)$. Such an analysis is performed for all possible reciprocal pairs. Here, in Figures 3(a) and 3(b), all analyses use the same $q$ and $\lambda^{*}$, namely $q=\frac{1}{2}$ and $\lambda^{*}=\frac{2}{3} \lambda \sin \alpha$ where $\alpha$ equals either $60^{\circ}$ or $120^{\circ}$. So $\lambda^{*}=\frac{1}{\sqrt{3}} \lambda$.

Other variants: In Figure 1(c), we return to the situation where only two types of seed exist, but each seed now has three emanating rays. Shading patterns show the reciprocal pairing, as described in the figure caption.

The E-rays are 'thick black' and these have one half the prevalence of the 'thick black' $60^{\circ}$ rays with which they are paired. So the $E$-rays can be analysed in generic form using $q=\frac{1}{3}$ and $\lambda^{*}=\lambda \sin 60^{\circ}$. To make the white system into the generic format, we reflect it about a vertical line; so now the horizontal white rays are growing east (having previously been $W$-rays that had twice the prevalence of their partner rays, also white). So the white $q=\frac{2}{3}$. The 'dashed black' $q$ equals either $\frac{1}{3}$ or $\frac{2}{3}$ depending on which direction of growth is asked to play the role of $E$-rays in the generic problem.

We also note that the generic analysis also applies to Figure 3(c), where there is only one type of seed, it growing two non-opposing rays. 
Further insight into Lemma 3: A referee's comment has led to this sub-section. Our earlier proof of Lemma 3 used an algebraic argument. Our seeds, though randomly positioned initially by a stationary Poisson process, were then fixed in position in both the original $\alpha=90^{\circ}$ case and the $\alpha \neq 90^{\circ}$ cases. Only the rays' growth-directions changed.

The referee has suggested a relocation of the seeds from their original positions. We describe this situation in the caption of Figure 4 and in the figure's sequel.

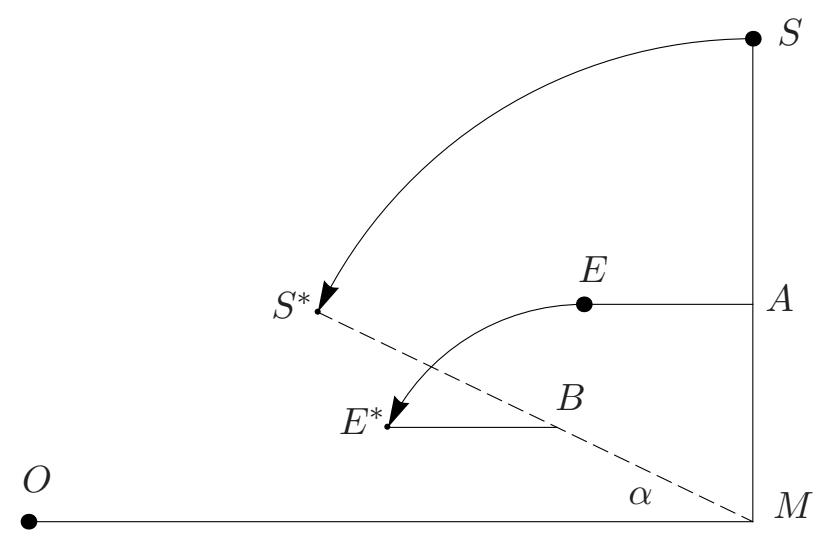

FIGURE 4. An east-growing ray starts at $O$ and a south-growing ray starts at $S$. In the absence of other rays, their paths in the $\alpha=90^{\circ}$ case would meet at $M$. When $\alpha$ is changed to the value shown in the figure, consider what happens if $S$ is moved around the circular arc to $S^{*}$ before ray growth commences. The ray then grows from $S^{*}$ toward $M$. Because the $\operatorname{arc}$ has centre $M,\left|S^{*} M\right|$ equals $|S M|$, and because $O$ is $(0,0)$ and $M$ is fixed, $|O M|$ is unchanged. So we see that (in the event that ray $S$ and ray $O$ actually reach $M$ ) which one blocks the other is unchanged. At $E$, however, is another east-growing ray; obviously it might block $S$ (at $A$ ). The role of this third ray, and any other relevant rays, is discussed further in the text.

Let seeds $S$ and $E$ have coordinates $\left(x_{1}, y_{1}\right)$ and $\left(x_{2}, y_{2}\right)$ respectively. The transformation that moves $S$ to $S^{*}$ is defined by $(x, y) \longrightarrow(x-y \cos \alpha, y \sin \alpha)$. Let us apply this transformation to all seeds ( $S, E, O$ and others) before ray-growth commences. We observe that $E$ is moved to $E^{*}$ and that $O$ doesn't move. Note that the arc on which $E$ moves has its centre at $\left(x_{2}, 0\right)$ on $O M$.

Now ray growth begins. The east-growing rays still grow eastward, but the erstwhile southgrowing rays now grow parallel to the dashed line $S^{*} M$. The seed at $E^{*}$ grows eastward and has the potential to meet the growth path of seed $S^{*}$ at $B$. Since $A$ is $\left(x_{1}, y_{2}\right)$ and $E^{*}$ is $\left(x_{2}-y_{2} \cos \alpha, y_{2} \sin \alpha\right)$ and $B$ is $\left(x_{1}-y_{2} \cos \alpha, y_{2} \sin \alpha\right)$, we see that $\left|E^{*} B\right|=|E A|$. Also, since $S^{*}$ is $\left(x_{1}-y_{1} \cos \alpha, y_{1} \sin \alpha\right),\left|S^{*} B\right|=|S A|$. So the respective distances travelled by $S$ and by $E$ to the point where their growth paths meet is the same before and after the transformation. The positions of $S$ and $E$ are general, so all pairs of seeds enjoy this preservation of growth distances. The transformation therefore does not affect which seeds block which - and each 
ray, in particular our test ray which starts at $O$, grows an identical length ray in the posttransformed situation as in the pre-transformed case.

Moreover, because the transformation is linear and invertible, the point process formed by applying the transformation to every seed is still a Poisson process (see the general issue of transformation in Baddeley [7]). It is also stationary with intensity $\lambda^{*}=\lambda \sin \alpha$. Stationarity can be proved by a measure-extension argument commencing with rectangles. The inverse image of any post-transformation rectangle in the plane having area $a$ is a parallelogram of area $a \sin \alpha$.

So Lemma 3 is proved in this clever way. Because this proof involves moved seeds, we recommend that the reader use our original concepts (involving fixed seeds) when assimilating the constructions behind Figure 1(c) and Figure 3, especially Figure 3(c). These constructions would be very difficult to understand with moving seeds.

\section{Acknowledgement}

We thank the two referees and a sub-editor for perceptive comments which have led to an improvement in the paper.

\section{REFERENCES}

[1] Gilbert, E. N. (1967) Surface Films of Needle-Shaped Crystals, In Applications of Undergraduate Mathematics in Engineering, B. Noble (editor), Macmillan, pp. 329-346.

[2] Mackisack, M. S. And Miles, R. E. (1996). Homogeneous Rectangular Tessellations. Adv. Appl. Prob. 28 993.

[3] Burridge, J., Cowan R. And Ma, I. (2013). Full and Half Gilbert Tessellations with Rectangular Cells. Adv. Appl. Prob. 45 1-19.

[4] Zuyev, S. (1999). Stopping Sets: Gamma-type results and hitting properties. Adv. Appl. Prob. 31 355-366.

[5] Cowan, R., Quine, M. And Zuyev, S. (2003). Decomposition of Gamma-distributed domains constructed from Poisson Point Processes. Adv. Appl. Prob. 35 56-69.

[6] Gradshteyn, I.S. And Ryzhik, I.M. (2007). Table of Integrals, Series, and Products, Academic Press.

[7] Baddeley, A. (2007) Spatial point processes and their application. In Stochastic Geometry, ed. W. Weil. Springer, Berlin. 